\title{
New solar angles and their corresponding tracking systems efficiency
}

\author{
M. Vătăşescu ${ }^{1}$, I. Vişa $^{1}$, D. Diaconescu ${ }^{1}$, I. Hermenean ${ }^{1}$, N. Creangă ${ }^{1}$ \\ ${ }^{1}$ Department of Product Design for Sustainable Development \\ Transilvania University of Braşov \\ B-ul Eroilor, 29, 500036 Braşov (România)
}

Phone/Fax number: +40-(268) 41.20.88,+40-(268) 41.05.25, e-mail: maria.vatasescu@unitbv.ro

\begin{abstract}
For increasing the tracking efficiency of a biaxial, bi-mobile photovoltaic (PV) system, there are identified and analyzed two new pairs of local and global angles, geometrically derived from the azimuth and equatorial configurations $[1,2]$. Simulations are done for the climatic and geographic region of Braşov, Romania, for one year duration. The results are showing that the new angles are able to provide a higher tracking efficiency as compared to their original configurations $[1,2]$. A relevant comparison is being drawn between six emerged cases.
\end{abstract}

\section{Key words}

Equatorial tracking system, azimuth (local) tracking system, solar direct radiation, direct tracking efficiency.

\section{Introduction}

The most significant parameter to evaluate the performance of a PV tracking system is the absolute direct tracking efficiency, representing the ratio between the energy of the direct solar radiation that falls normally on a PV surface and the energy of the available direct solar radiation [3]; a biaxial PV tracker is usually efficient if its direct tracking efficiency is over $97 \%$.

The literature [3] presents two terrestrial systems which are describing the solar ray orientation (see Fig.1): the equatorial system $\operatorname{OXYZ}(\delta, \omega)$ and the azimuth system $\mathrm{QX}_{0} \mathrm{Y}_{0} \mathrm{Z}_{0}(\alpha, \psi)$; from these angular pairs were derived the classic tracking open linkages (see Fig.2b and $3 b$ ).

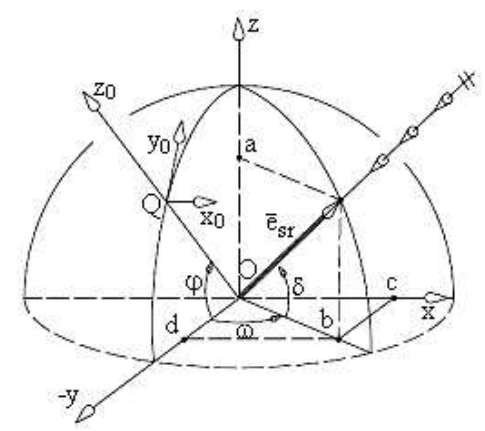

Fig.1 Equatorial system OXYZ (with the solar ray angles: hour angle $\omega$ and declination $\delta$ ) and azimuth system $\mathrm{QX}_{0} \mathrm{Y}_{0} \mathrm{Z}_{0}$ (with the solar ray angles: azimuth $\psi$ and elevation $\alpha$ )
The mechanical structure of a tracking system has to follow the Sun path so that the maximum direct tracking efficiency is obtained with minimum energetic and technical resources. In most cases, this implies using linear actuators instead of rotating ones (which are far more expensive). Usually, linear actuators require reduced PV angular strokes $\left(\Delta \omega^{*}\right.$ and $\left.\Delta \psi^{*}\right)$ as compared to sun ray daily angular strokes $(\Delta \omega$ and $\Delta \psi)$. As a consequence, it becomes necessary the analysis of the direct tracking efficiency when considering reduced angular strokes for both the equatorial and the azimuth tracking systems.

Daily and elevation sun-ray orientation can be described also by another two angular pairs drawn in each of the two systems: $\mathrm{OXYZ}$ and $\mathrm{QX}_{0} \mathrm{Y}_{0} \mathrm{Z}_{0}$, as presented in figures $2 \mathrm{a}$ and $3 \mathrm{a}[1,2]$; from these new angular pairs, four new tracking open linkages are proposed, as presented in figures $2 \mathrm{c}, 2 \mathrm{~d}, 3 \mathrm{c}$ and $3 \mathrm{~d}$.

The aim of this paper is to model the new proposed angular pairs (as against the primary angles: $\omega$ and $\delta$ ) and to analyze by comparison, trough numerical simulations, their direct tracking efficiencies.

As a consequence, the classical and the new proposed tracking open linkages are compared when: a) the daily and elevation PV angular strokes are equal to homologous sun-ray angular strokes and b) the daily PV angular strokes are smaller than the daily sun-ray strokes. The numerical simulations are performed in the premise that the daily and elevation angular displacements of the PV-module are done as hourly steps.

\section{The geometrical modeling of the solar angular pairs}

Instead of the classic angular pairs $(\delta, \omega)$ and $(\alpha, \psi)$, the sun ray orientation can also be described by another two angular pairs: $(\omega 1, \delta 1)$ and $(\omega 2, \delta 2)$ in the equatorial system OXYZ (see Fig.2a) and $(\psi 1, \alpha 1)$ and $(\psi 2, \alpha 2)$, in the azimuth system $\mathrm{QX}_{0} \mathrm{Y}_{0} \mathrm{Z}_{0}$ (see Fig.3a) [1, 2]. The mathematic expressions for the new angles are established, by geometrical calculus, as presented in figures $2 \mathrm{a}$ and $3 \mathrm{a}$, as opposed to the classical angles. 
a)

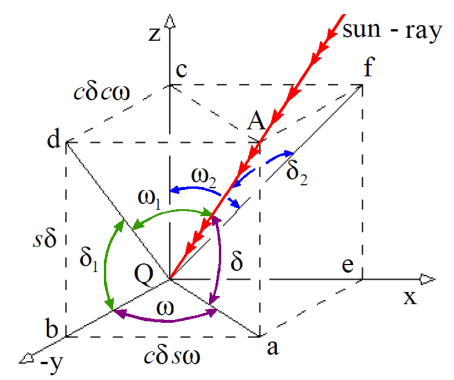

b)

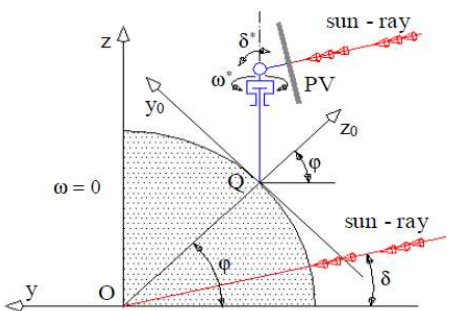

c)

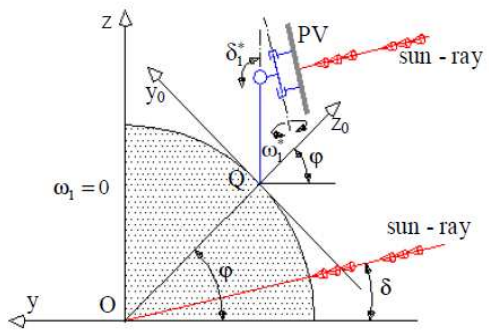

d)

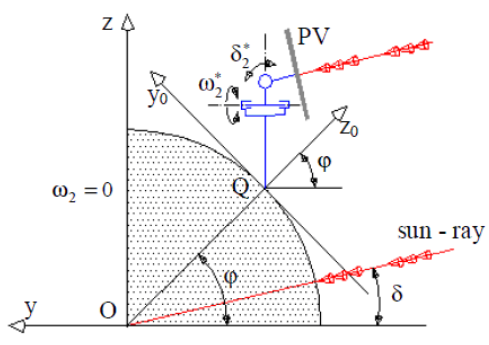

Fig. 2 a) The angular pairs $\boldsymbol{\delta}, \boldsymbol{\omega} ; \boldsymbol{\delta 1}, \boldsymbol{\omega} \mathbf{1}$ and $\boldsymbol{\delta} \mathbf{2}, \boldsymbol{\omega} \mathbf{2}$, which describe the sun ray orientation in the global system OXYZ; b), c) and d) - the corresponding tracking open linkages and their positions relative to the Earth, at noon

The expressions for $\omega$ and $\delta$, on system OXYZ, are:

$$
\begin{gathered}
\omega=15^{\circ}(12-T) \\
\delta=23,45^{\circ} \sin \left(\frac{360^{\circ}(N-80)}{365}\right)
\end{gathered}
$$

in which $\mathrm{T}$ represents the solar time value and $\mathrm{N}$ represents the number of the current day [3].

For the other angles plotted in figure $2 \mathrm{a}$ the mathematic expressions are:

$$
\begin{gathered}
\omega 1=\arcsin (\cos \delta \cdot \sin \omega) \\
\delta 1=\arcsin \left(\frac{\sin \delta}{\sqrt{1-(\cos \delta \cdot \cos \omega)^{2}}}\right) \\
\omega 2=\arccos \left(\frac{\sin \delta}{\sqrt{1-(\cos \delta \cdot \cos \omega)^{2}}}\right)(\operatorname{sgn} \omega) \\
\delta 2=\arcsin (\cos \delta \cdot \cos \omega)
\end{gathered}
$$

a)

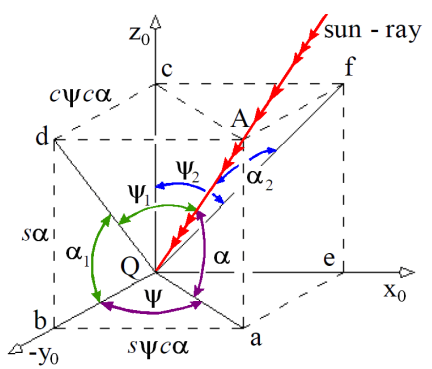

b)

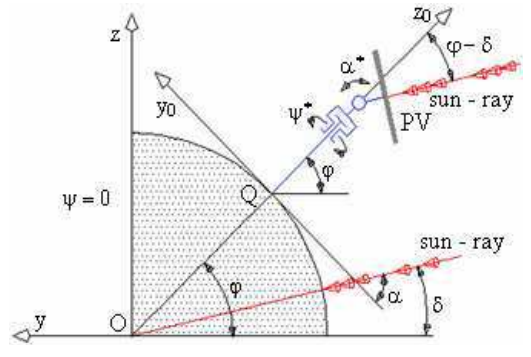

c)

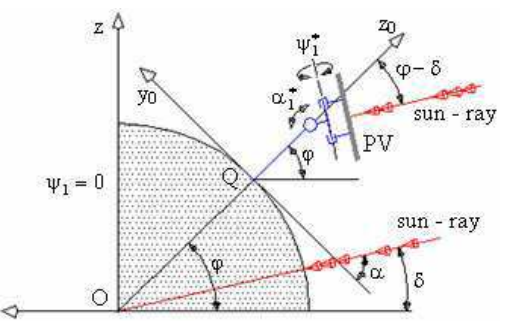

d)

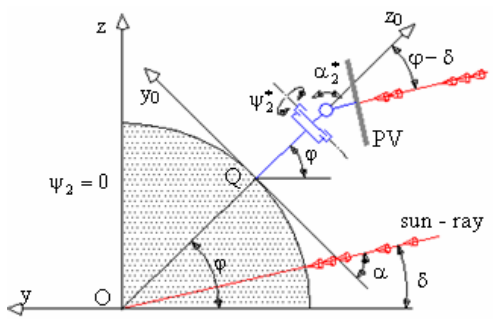

Fig. 3 a) The angular pairs $\alpha, \boldsymbol{\psi} ; \boldsymbol{\alpha 1}, \boldsymbol{\psi 1}$ and $\boldsymbol{\alpha 2}, \boldsymbol{\psi 2}$, which describe the sunray orientation in the local system $\mathrm{QX}_{0} \mathrm{Y}_{0} \mathrm{Z}_{0}$; b), c) and d) - the corresponding tracking open linkages and their positions relative to the Earth, at noon

The expressions for $\alpha$ and $\psi$, on system $\mathrm{OX}_{\mathrm{o}} \mathrm{Y}_{\mathrm{o}} \mathrm{Z}_{\mathrm{o}}$, are:

$$
\begin{aligned}
& \alpha=\arcsin (\cos \varphi \cos \delta \cos \omega+\sin \varphi \sin \delta) \\
& \psi=(\operatorname{sgn} \omega) \arccos \left(\frac{\sin \varphi \sin \alpha-\sin \delta}{-\cos \alpha \cos \varphi}\right)
\end{aligned}
$$

In which $\varphi=45,65^{\circ}$ lat. $\mathrm{N}$

For the other angles plotted in figure $3 \mathrm{a}$ the mathematic expressions are:

$$
\begin{gathered}
\alpha 1=\arccos \left(\frac{\cos \alpha \cdot \cos \psi}{\sqrt{1-(\cos \alpha \cdot \sin \psi)^{2}}}\right) \\
\psi 1=\arcsin (\cos \alpha \cdot \sin \psi) \\
\alpha 2=\arcsin (\cos \alpha \cdot \cos \psi) \\
\psi 2=\arccos \left(\frac{\sin \alpha}{\sqrt{1-(\cos \alpha \cdot \cos \psi)^{2}}}\right)(\operatorname{sgn} \psi)
\end{gathered}
$$


The real angular strokes for the six sunray angular pairs (for geographic region of Braşov, Romania) are established by simulating their mathematic expressions during the main, representative solar moments of the year: the spring/autumn equinox, the summer and winter solstice (see Table I). The angular stroke of any solar angle is obtained as difference between the extreme values of the considered solar angle (see Table II)

Table I. Annual extreme values of the solar angular pairs afferent to the six tracking open linkages from Fig.2 and 3

\begin{tabular}{|c|c|c|c|c|c|}
\hline \multicolumn{3}{|c|}{ The AZIMUTH systems } & \multicolumn{3}{|c|}{$\begin{array}{c}\text { The EQUATORIAL } \\
\text { systems }\end{array}$} \\
\hline \multicolumn{2}{|c|}{ Morning/Evening } & Noon & Mor & ing/Evening & Noon \\
\hline \multicolumn{6}{|c|}{ Spring/Autumn Equinox } \\
\hline$\alpha$ & $0^{\circ}$ & $45^{\circ}$ & $\delta$ & $0^{\circ}$ & $0^{\circ}$ \\
\hline$\psi$ & $90^{\circ} /-90^{\circ}$ & $0^{\circ}$ & $\omega$ & $90^{\circ} /-90^{\circ}$ & $0^{\circ}$ \\
\hline$\alpha 1$ & $45^{\circ}$ & $45^{\circ}$ & $\delta 1$ & $0^{\circ}$ & $0^{\circ}$ \\
\hline$\psi 1$ & $90^{\circ} /-90^{\circ}$ & $0^{\circ}$ & $\omega 1$ & $90^{\circ} /-90^{\circ}$ & $0^{\circ}$ \\
\hline$\alpha 2$ & $0^{\circ}$ & $46^{\circ}$ & $\delta 2$ & $0^{\circ}$ & $90^{\circ}$ \\
\hline$\psi 2$ & $90^{\circ} /-90^{\circ}$ & $0^{\circ}$ & $\omega 2$ & $-90^{\circ}$ & $-90^{\circ}$ \\
\hline \multicolumn{6}{|c|}{ Summer Solstice } \\
\hline$\alpha$ & $0^{\circ}$ & $68^{\circ}$ & $\delta$ & $23,45^{\circ}$ & $23,45^{\circ}$ \\
\hline$\psi$ & $125^{\circ} /-125^{\circ}$ & $0^{\circ}$ & $\omega$ & $117^{\circ} /-117^{\circ}$ & $0^{\circ}$ \\
\hline$\alpha 1$ & $180^{\circ}$ & $66^{\circ}$ & $\delta 1$ & $136^{\circ}$ & $23^{\circ}$ \\
\hline$\psi 1$ & $56^{\circ} /-56^{\circ}$ & $0^{\circ}$ & $\omega 1$ & $90^{\circ} /-90^{\circ}$ & $0^{\circ}$ \\
\hline$\alpha 2$ & $-34^{\circ}$ & $24^{\circ}$ & $\delta 2$ & $-24^{\circ}$ & $67^{\circ}$ \\
\hline$\psi 2$ & $90^{\circ} /-90^{\circ}$ & $0^{\circ}$ & $\omega 2$ & $65^{\circ} /-65^{\circ}$ & $0^{\circ}$ \\
\hline \multicolumn{6}{|c|}{ Winter Solstice } \\
\hline$\alpha$ & $0^{\circ}$ & $21^{\circ}$ & $\delta$ & $-23,45^{\circ}$ & $-23,45^{\circ}$ \\
\hline$\psi$ & $56^{\circ} /-56^{\circ}$ & $0^{\circ}$ & $\omega$ & $64^{\circ} /-64^{\circ}$ & $0^{\circ}$ \\
\hline$\alpha 1$ & $0^{\circ}$ & $21^{\circ}$ & $\delta 1$ & $-45^{\circ}$ & $-23^{\circ}$ \\
\hline$\psi 1$ & $56^{\circ} /-56^{\circ}$ & $0^{\circ}$ & $\omega 1$ & $56^{\circ} /-56^{\circ}$ & $0^{\circ}$ \\
\hline$\alpha 2$ & $35^{\circ}$ & $70^{\circ}$ & 82 & $25^{\circ}$ & $67^{\circ}$ \\
\hline$\psi 2$ & $90^{\circ} /-90^{\circ}$ & $0^{\circ}$ & $\omega 2$ & $116^{\circ} /-116^{\circ}$ & $\pm 180^{\circ}$ \\
\hline
\end{tabular}

An hourly tracking program is simulated for each PV daily angular stroke, in two situations: a) when the PV daily angular stroke is equal to the homologous sunray stroke; b) when the PV daily angular stroke is smaller than the sunray stroke. The smaller daily angular strokes are presented in Table II.

The PV angles respect the notations of their homologues sunray angles, additionally marked with a star.

Table II. Reduced and complete daily angular strokes for the tracking open linkages of equatorial and azimuth type

\begin{tabular}{|c|c|c|c|c|c|c|c|}
\hline \multicolumn{8}{|c|}{ Daily PV angular strokes } \\
\hline \multicolumn{2}{|c|}{ Complete } & \multicolumn{6}{|c|}{ Reduced } \\
\hline$\Delta \omega^{*}$ & $240^{\circ}$ & $120^{\circ}$ & $150^{\circ}$ & $180^{\circ}$ & $210^{\circ}$ & & \\
\hline$\Delta \omega 1^{*}$ & $180^{\circ}$ & $120^{\circ}$ & $150^{\circ}$ & & & & \\
\hline$\Delta \omega D^{*}$ & $360^{\circ}$ & $120^{\circ}$ & $150^{\circ}$ & $180^{\circ}$ & $210^{\circ}$ & $240^{\circ}$ & $270^{\circ}$ \\
\hline & & & & & & $300^{\circ}$ & $330^{\circ}$ \\
\hline$\Delta \psi^{*}$ & $240^{\circ}$ & $120^{\circ}$ & $150^{\circ}$ & $180^{\circ}$ & $210^{\circ}$ & & \\
\hline$\Delta \psi 1^{*}$ & $180^{\circ}$ & $120^{\circ}$ & $150^{\circ}$ & & & & \\
\hline$\Delta \psi 2 *$ & $180^{\circ}$ & $120^{\circ}$ & $150^{\circ}$ & & & & \\
\hline
\end{tabular}

In order to determine the direct tracking efficiencies for each of the six tracking open linkages (see Fig. 2b, 2c, $2 \mathrm{~d}, 3 \mathrm{~b}, 3 \mathrm{c}$ and $3 \mathrm{~d}$ ) is required the modeling of the incidence angles and of the direct received radiation for each six configurations.

\section{Incidence angles and solar direct radiation modeling}

As the incidence angle is measured between the solar ray unit vector and the normal unit vector to the PV surface, the solar ray unit vectors, are determined as follows:

- For the solar angular pairs of equatorial type (Fig.2a):

$$
\begin{gathered}
e_{s r}(\delta, \omega)=\left[\begin{array}{c}
\cos \delta \sin \omega \\
-\cos \delta \cos \omega \\
\sin \delta
\end{array}\right]_{X Y Z}, \quad e_{s r}(\delta 1, \omega 1)=\left[\begin{array}{c}
\sin \omega 1 \\
-\cos \delta 1 \cos \omega 1 \\
\sin \delta 1 \cos \omega 1
\end{array}\right]_{X Y Z} \\
e_{s r}(\delta 2, \omega 1)=\left[\begin{array}{c}
\cos \delta 2 \sin \omega 2 \\
-\sin \delta 2 \\
\cos \delta 2 \cos \omega 2
\end{array}\right]_{X Y Z} ;(7),\left(7^{\prime}\right),\left(7^{\prime},\right)
\end{gathered}
$$

these expressions are also valid for the PV normal unit vectors, but with stars attached at the angle notations; therefore, the corresponding incidence angles become:

$$
\begin{gathered}
v_{e q}=\arccos \left[\sin \delta \sin \delta *+\cos \delta \cos \delta * \cos \left(\omega-\omega^{*}\right)\right] \\
v 1_{e q}=\arccos \left[\sin \omega 1 \sin \omega 1^{*}+\cos \omega 1 \cos \omega 1^{*} \cos \left(\delta 1-\delta 1^{*}\right)\right] \\
v 2_{e q}=\arccos \left[\sin \delta 2 \sin \delta 2 *+\cos \delta 2 \cos \delta 2 * \cos \left(\omega 2-\omega 2^{*}\right)\right]
\end{gathered}
$$

- For the solar angular pairs of azimuth type (Fig.3a), the following expressions are obtained similarly:

$$
\begin{aligned}
& e_{s r}(\alpha, \psi)=\left[\begin{array}{c}
\cos \alpha \sin \psi \\
-\cos \alpha \cos \psi \\
\sin \alpha
\end{array}\right]_{X_{0} Y_{0} Z_{0}} e_{s r}(\alpha 1, \psi 1)=\left[\begin{array}{c}
\sin \psi 1 \\
-\cos \alpha 1 \cos \psi 1 \\
\sin \alpha 1 \cos \psi 1
\end{array}\right]_{X_{0} Y_{0} Z_{0}} \\
& e_{s r}(\alpha 2, \psi 2)=\left[\begin{array}{c}
\cos \alpha 2 \sin \psi 2 \\
-\sin \alpha 2 \\
\cos \alpha 2 \cos \psi 2
\end{array}\right]_{X_{0} Y_{0} Z_{0}} ; \quad(9),\left(9^{\prime}\right),\left(9^{\prime \prime}\right) \\
& v_{a z}=\arccos \left[\sin \alpha \sin \alpha^{*}+\cos \alpha \cos \alpha^{*} \cos \left(\psi-\psi^{*}\right)\right] \\
& v 1_{a z}=\arccos \left[\sin \psi 1 \sin \psi 1^{*}+\cos \psi 1 \cos \psi 1^{*} \cos \left(\alpha 1-\alpha 1^{*}\right)\right] \\
& v 2_{a z}=\arccos \left[\sin \alpha 2 \sin \alpha 2^{*}+\cos \alpha 2 \cos \alpha 2^{*} \cos \left(\psi 2-\psi 2^{*}\right)\right]
\end{aligned}
$$

The available solar radiation is determined as [3]:

$$
\begin{gathered}
B_{0}=1367 \cdot\left[1+0,0334 \cdot \cos \left(0,9856^{\circ} N-2,72\right)\right]\left[W / m^{2}\right] \\
B s=B_{0} \exp \left(-\frac{T r}{0,9+9,4 \sin \alpha}\right)\left[W / m^{2}\right]
\end{gathered}
$$

and the received solar radiation falling normal on the PV $[1,2]$, according to the chosen system, is:

$$
B_{P V k}=B_{s} \cdot \cos v_{k}, \mathrm{k}=\{e q, e q 1, e q 2, a z, a z 1, a z 2\}
$$

These determinations are used in the numerical simulations of the direct tracking efficiencies for each of the six tracking open linkages, considering an ideal permanent clear sky [3]. 


\section{The comparative analysis by numerical simulation}

To simplify the calculus of the annual direct tracking efficiency, the year is divided in four seasons and for each there are considered the middle and the delimiting days. Therefore the numerical simulations are done during the following twelve days of the year: day 355 the Winter Solstice, day 80 - the Spring Equinox, day 172 - the Summer Solstice, day 266 - the Autumn Equinox, days 60 and 105 - delimiting the Spring season; days 106 and 237 - delimiting the Summer season, days 238 and 280 - delimiting the Autumn season and days 281 and 60 - delimiting the Winter season [4].

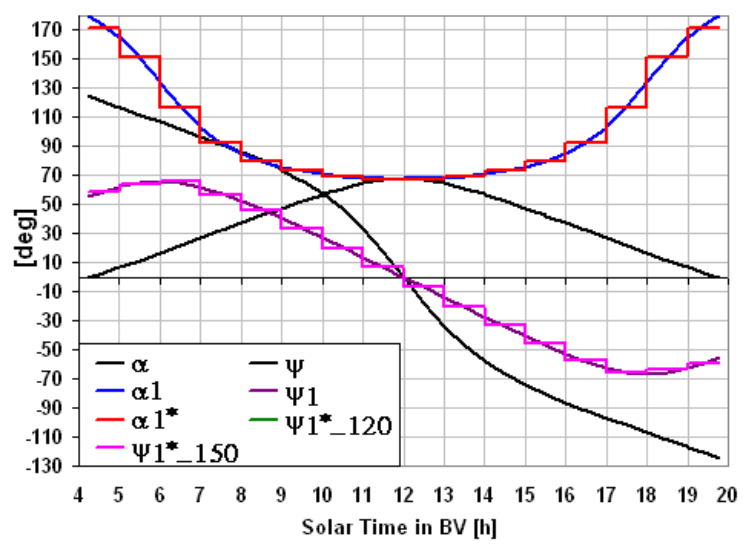

a)

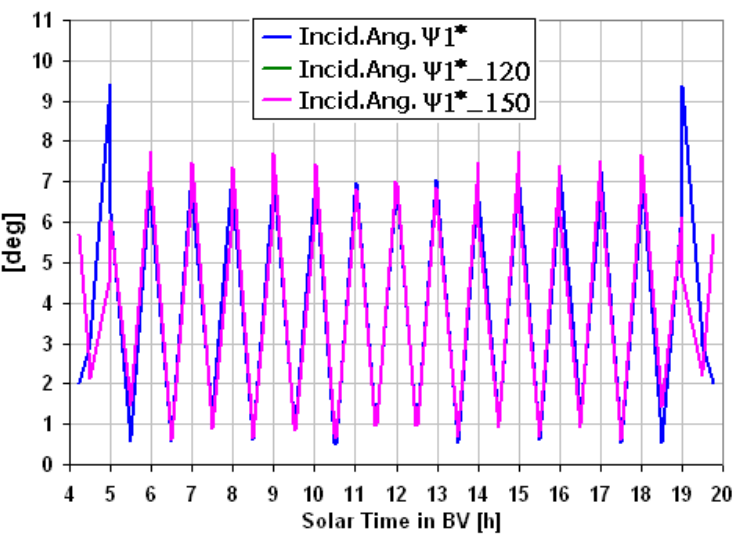

b)

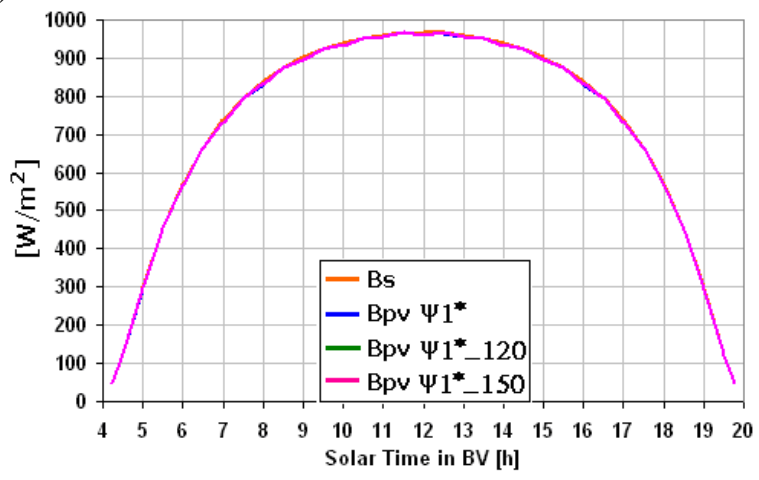

Fig. 4 Numerical simulations on the Summer Solstice day for: a) the sun angles: $\alpha, \alpha 1, \psi, \psi 1$; the PV-elevation $\alpha 1 *$ and the PV-azimuth reduced angular strokes $\psi 1 * 120$ (with an angular stroke of $120^{\circ}$ ) and $\psi 1 * 150$ (with an angular stroke of $150^{\circ}$ ); b) the correspondent incidence angles; c) the available and the correspondent solar direct received radiations.
In each of these days, six cases were simulated, presenting the behavior of a bi-axial, bi-mobile tracking open linkage during a year:

- Three equatorial tracking programs with an angular adjustment at every hour, covering the entire sunray daily angular strokes $\Delta \omega, \Delta \omega 1, \Delta \omega 2$;

- Three sets of equatorial tracking programs with the angular adjustment at every hour, for a number of reduced daily angular strokes: $\Delta \omega^{*}, \Delta \omega 1^{*}, \Delta \omega 2^{*}$;

- Three azimuth tracking programs with an angular adjustment at every hour, covering the entire sunray daily angular strokes: $\Delta \psi, \Delta \psi 1, \Delta \psi 2$;

- Three sets of azimuth tracking programs with the angular adjustment at every hour, for a number of reduced daily angular strokes: covering the entire sunray daily angular strokes $\Delta \psi, \Delta \psi 1, \Delta \psi 2$.

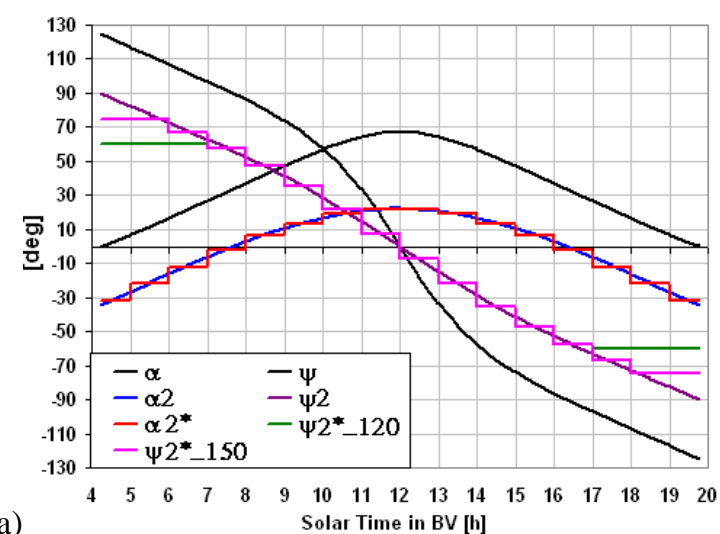

a)
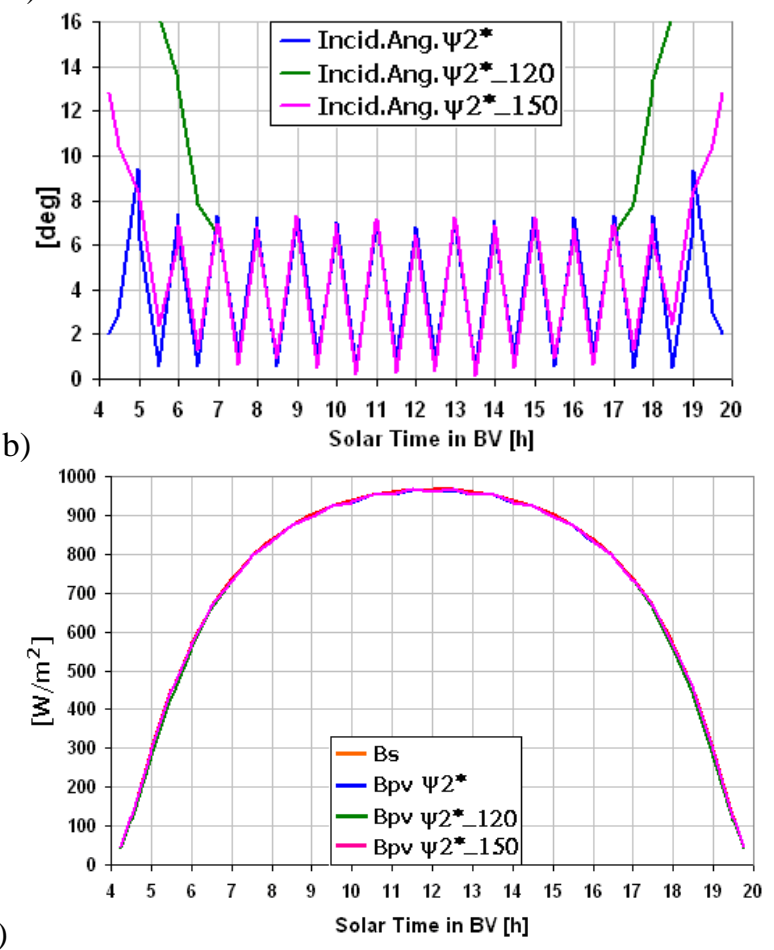

Fig. 5 Numerical simulations on the Summer Solstice day for: a) the sun angles: $\alpha, \alpha 2, \psi, \psi 2$; the PV-elevation $\alpha 2 *$ and the $\mathrm{PV}$-azimuth reduced angular strokes $\psi 2 * 120$ (with an angular stroke of $120^{\circ}$ ) and $\psi 2 * 150$ (with an angular stroke of $150^{\circ}$ ); b) the correspondent incidence angles; c) the available and the correspondent solar direct received radiations. 
To analyze the energetic behavior of the six biaxial, bimobile open linkages tracking systems (see Fig. 2b, 2c, $2 \mathrm{~d}, 3 \mathrm{~b}, 3 \mathrm{c}, 3 \mathrm{~d})$, their daily angular strokes $\left(\omega^{*}, \omega 1^{*}, \omega 2^{*}\right.$, $\left.\psi^{*}, \psi 1^{*}, \psi 2^{*}\right)$ are diminished each with $30^{\circ}, 60^{\circ}, 90^{\circ}$, $120^{\circ}, 180^{\circ}$ and $200^{\circ}$, where possible (see Table II).

The reduced strokes can be noticed in figures $5 \mathrm{a}, 6 \mathrm{a}$ and $7 \mathrm{a}$, while in figure $4 \mathrm{a}$ it does not appear as the $\psi 1$ maximum stroke is $125^{\circ}$, so $\psi 1 *$ does not require reduction. The two new azimuth configurations, having the reduced daily angular strokes: $\psi 1^{*} \_120, \psi^{*} \_180$, $\psi 2 * \_120, \psi^{*} 2 \_150, \psi 2 * \_180$, are presented in figures 4 and 5 , in the summer solstice day so the differences between the solar angles $(\alpha, \psi, \alpha 1, \psi 1)$ and $(\alpha, \psi, \alpha 2$, $\psi 2$ ) can be observed. In this case the negative values for the sun elevation $\alpha 1$ are not abnormal, but are due to the sunrise and sunset positions behind ZQX plane (see Fig. 2a). In $4 \mathrm{a}$, can be noticed the resemblance between $\alpha 1$ and $\psi 1$ curves and $\delta$ and $\gamma$ variations from a pseudoequatorial local system [5].

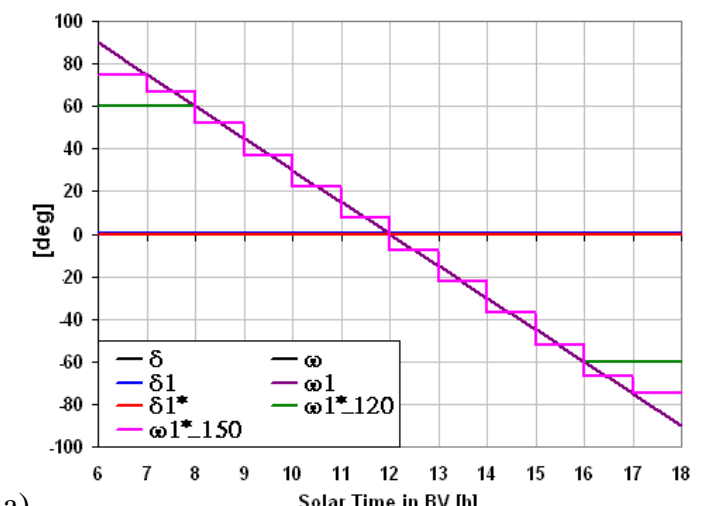

a)

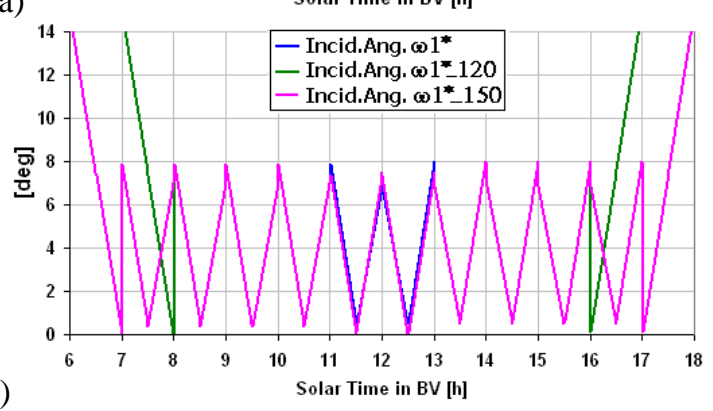

b)

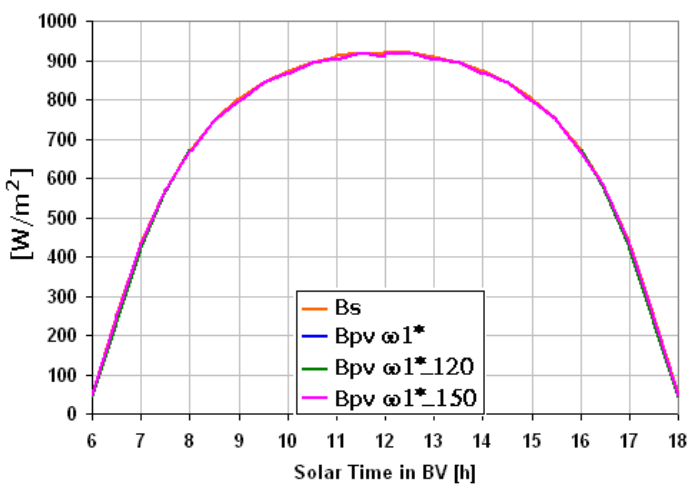

c)

Fig. 6 Numerical simulations on the Spring Equinox day for: a) the sun angles: $\delta, \delta 1, \omega, \omega 1$; the PV-elevation: $\delta 1 *$ and the PV-hour angle reduced stoke $\omega 1 * 120$ (with an angular stroke of $120^{\circ}$ ) and $\omega 1^{*} 150$ (with an angular stroke of $150^{\circ}$ );

b) the correspondent incidence angles; c) the available and the correspondent solar direct received radiations.
The equatorial system 1 requires the maximum number of reduced PV daily strokes in spring and autumn, each represented by the spring equinox day, as presented in figure 6.

Likewise, equatorial system 2 requires the maximum number of reduced PV daily strokes in winter, simulated in the winter solstice day, as presented in figure 7. This particular case encounters the highest deviations for the incidence angle (see Fig.7b) which lead to low receiving direct solar radiations (see Fig. 7c)

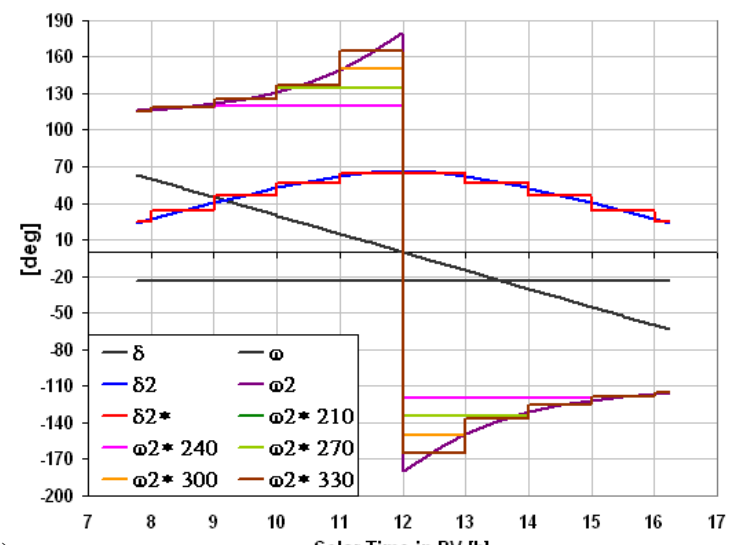

a)

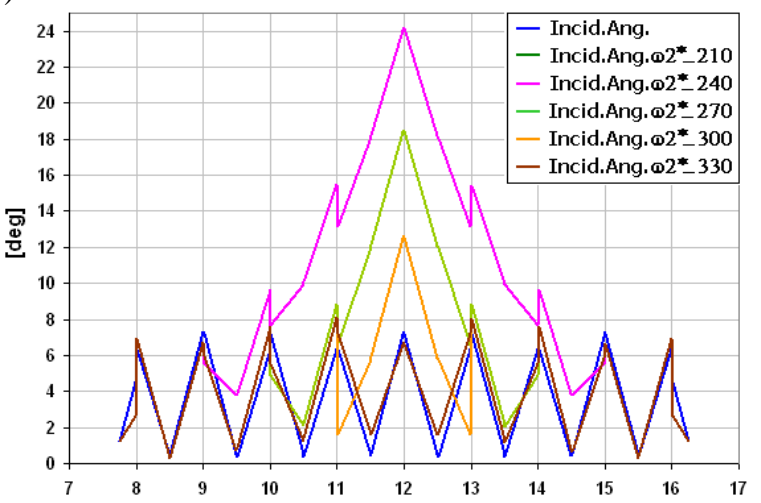

b) Solar Time in BV [h]

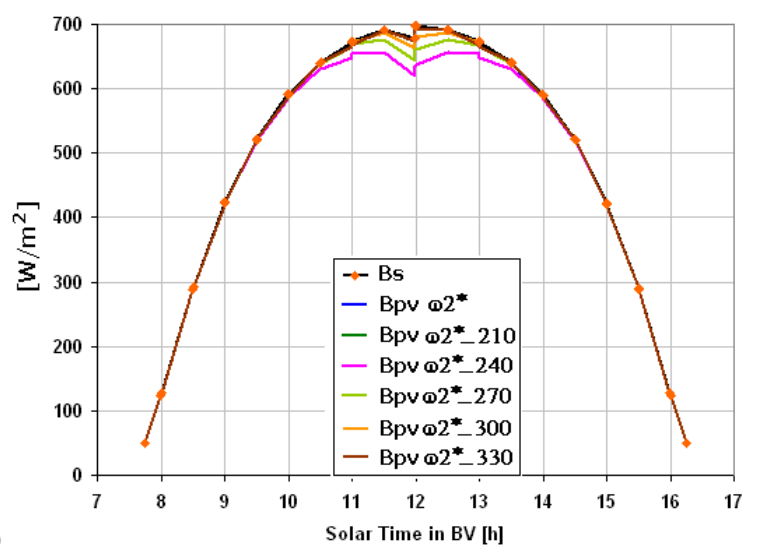

Fig. 7 Numerical simulations on the Winter Solstice day for: a) the sun angles: $\delta, \delta 2, \omega, \omega 2$; PV-elevation: $\delta 2 *$ and the PVhour angle reduced stroke $\omega 2 * 120$ (with an angular stroke of $120^{\circ}$ ), $\omega 2 * 150$ (with an angular stroke of $150^{\circ}$ ); $\omega 2 * 180$ (with an angular stroke of $180^{\circ}$ ); $\omega 2 * 210$ (with an angular stroke of $210^{\circ}$ ); $\omega 2 * 240$ (with an angular stroke of $240^{\circ}$ ); $\omega 2 * 270$ (with an angular stroke of $270^{\circ}$ ); $\omega 2 * 300$ (with an angular stroke of $300^{\circ}$ ); $\omega 2 * 330$ (with an angular stroke of $330^{\circ}$ );

b) the correspondent incidence angles; c) the available and the correspondent solar direct received radiations. 
Daily solar energetic gain, for the climatic and geographic region of Brasov, Romania is estimated by calculating the surface under the solar radiation curve, then multiplying the obtained figure with the specific value FCC (Factor of Cloud Crossing). The FCC represents a ratio between the energy of the solar direct radiation under the clouds and the energy of the solar direct radiation above the clouds layer [4]. For each of the twelve representative days of the year, is calculated:

$$
E_{k}=F C C\left(\int B_{P V k} d t\right), \mathrm{k}=\{e q, e q 1, e q 2, a z, a z 1, a z 2\} .
$$

The seasonal solar energetic gain is calculated as average value between the daily energetic gain, considering the middle and delimiting season days. Likewise, the annual solar energetic gain is evaluated as an average of the four seasonal solar energetic gain values, previously calculated.

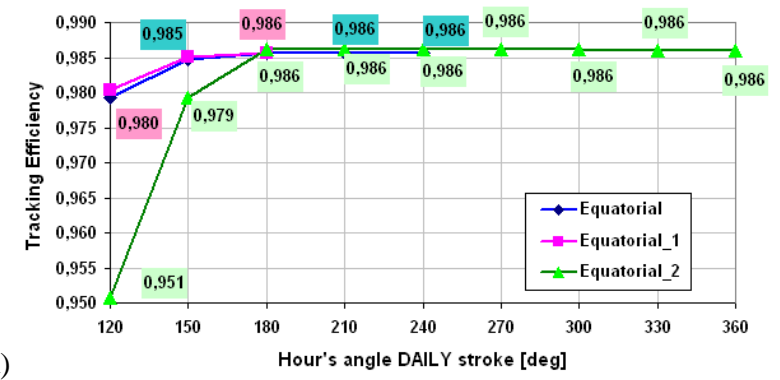

a)

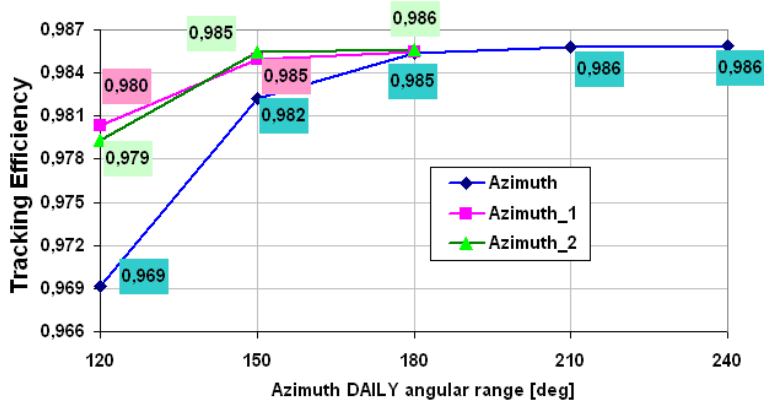

b)

Fig. 8 Annual tracking efficiency specific to each reduced daily tracking stroke of: a) equatorial type, b) azimuth type

Table III. The initial and the most reduced daily strokes and their corresponding direct tracking efficiencies for each equatorial and azimuth type.

\begin{tabular}{|c|c|c|c|c|c|c|}
\hline & $\Delta \omega^{*}$ & $\Delta \omega 1^{*}$ & $\Delta \omega 2^{*}$ & $\Delta \psi^{*}$ & $\Delta \psi 1^{*}$ & $\Delta \psi 2^{*}$ \\
\hline \multirow{3}{*}{ Complete } & $240^{\circ}$ & $180^{\circ}$ & $360^{\circ}$ & $240^{\circ}$ & $180^{\circ}$ & $180^{\circ}$ \\
\cline { 2 - 7 } & \multicolumn{7}{|c|}{ Direct tracking efficiency [\%] } \\
\cline { 2 - 7 } & 98,5 & 99,2 & 98,6 & 98,4 & 98,5 & 98,6 \\
\hline \multirow{3}{*}{ Reduced } & $120^{\circ}$ & $120^{\circ}$ & $180^{\circ}$ & $150^{\circ}$ & $120^{\circ}$ & $120^{\circ}$ \\
\cline { 2 - 7 } & \multicolumn{7}{|c|}{ Direct tracking efficiency [\%] } \\
\cline { 2 - 7 } & 98,5 & 98,5 & 98,6 & 98,2 & 98 & 98,5 \\
\hline
\end{tabular}

The annual solar energetic gains calculated for each reduced daily angular stroke, as presented in figure 8 , show that for attaining a tracking efficiency upwards of $97 \%$, the hourly angular range $\Delta \omega^{*}$ and $\Delta \omega 1^{*}$ can be diminished at $120^{\circ}$, while the $\Delta \omega 2^{*}$ should be set at $180^{\circ}$. In what concerns the azimuth systems, the angular stroke
$\Delta \psi^{*}$ should not be lowered under $150^{\circ}$, while $\Delta \psi 1^{*}$ and $\Delta \psi 2^{*}$ can be diminished to $120^{\circ}$ and still surpass the minimum imposed usable tracking efficiency of $97 \%$.

\section{Conclusions}

1. Sunray displacement can be described by three angular pairs in OXYZ global system and another three angular pairs in $\mathrm{QX}_{0} \mathrm{Y}_{0} \mathrm{Z}_{0}$ local system. From these configurations are derived six tracking open linkages, two of them being already known and used, namely: Equatorial and Azimuth tracking open linkages.

2. Although, the three equatorial tracking open linkages have the same mechanical structure as the three azimuth linkages, they are distinguish in between by their relative position to Earth; moreover, Equatorial 1 and Azimuth 1 linkages are in fact identical and are mentioned in the literature as pseudo-equatorial model, but they can also be denominated pseudo-azimuth model.

3. A comparative analysis between the direct tracking efficiencies show that all tracking open linkages allow an important reduction of the daily angular stroke with a negligible reduction of the tracking efficiency.

4. Considering the direct tracking efficiencies and their construction features, the Equatorial $1 \equiv$ Azimuth 1 . The Equatorial linkages are suited for the small and medium PV platforms, while Azimuth and Azimuth 2 linkages are suited for large PV platforms; from the last two, Azimuth linkage is preferred due to its construction advantages, though its daily angular stroke is larger.

\section{Acknowledgement:}

This paper is supported by the Sectoral Operational Programme Human Resources Development (SOP HRD), financed from the European Social Fund and by the Romanian Government under the contract number POSDRU/6/1.5/S/6".

\section{References:}

[1] Diaconescu, D. and Vătăşescu, M., "Two New Pairs of Local Solar Angles and Their Corresponding Tracking Systems", in Bulletin of the Transilvania University of Braşov, Vol. 1 (50) 114 - 2008, Series I, pp. 113-120.

[2] Vişa, I. and Hermenean, I., "On the Equatorial Type Systems Used in CPV Tracking", in Bulletin of the Transilvania University of Braşov, Vol. 1 (50) 114 - 2008, Series I, pp. 175-182.

[3] Meliss, M., "Regenerative Energie-quellen Praktikum", Springer, Berlin (1997), pp. 5-7.

[4] Diaconescu, D. et al., "Clouds Influence o the solar Radiation for a Mountain Location"; In: Environmental Engineering and Management Journal, july/august 2009, Vol.8, No.4, pp: 849-853; ISSN 1582 - 9596.

[5] Diaconescu, D., Visa, I., Burduhos, B.: "On the Received Direct Solar Radiance of the PV Panel Orientated by Pseudoequatorial Tracker", COMEC - The 2nd International Conference "Computational Mechanics and Virtual Engineering", Brasov, Romania, 11 - 13 Octombrie 2007, ISBN 978-973-598-117-4, pg. 43-48. 